\title{
Types, differences and prognosis of colonic polyps
}

\author{
Rahmathulla Safiyul Rahman ${ }^{1 *}$, Ali Saeed Alharbi ${ }^{2}$, Bassam Ahmed Basaben $^{3}$, \\ Ahmad Adnan Alsalman ${ }^{4}$, Anas Sulaiman Aljohani ${ }^{5}$, Bushra Abdulrahman Banafea ${ }^{6}$, \\ Hadeel Saad Aldhfyan ${ }^{7}$, Amna Farouk Ammar ${ }^{8}$, Wesam Mohammed Areeshi', \\ Hammam Khalid Alhussain ${ }^{10}$, Turki Bandar Alotaibi ${ }^{11}$
}

\author{
${ }^{1}$ Department of Internal Medicine, Sameera Medical Center, Jeddah, Saudi Arabia \\ ${ }^{2}$ College of Medicine, Medical University of Lodz, Lodz, Poland \\ ${ }^{3}$ College of Medicine, King Abdulaziz University in Rabigh, Rabigh, Saudi Arabia \\ ${ }^{4}$ College of Medicine, Jagiellonian University, Kraków, Poland \\ ${ }^{5}$ College of Medicine, Taibah University, Medina, Saudi Arabia \\ ${ }^{6}$ College of Medicine, Batterjee Medical College, Jeddah, Saudi Arabia \\ ${ }^{7}$ College of Medicine, Alfaisal University, Riyadh, Saudi Arabia \\ ${ }^{8}$ Department of Internal Medicine, King Fahad General Hospital, Jeddah, Saudi Arabia \\ ${ }^{9}$ College of Medicine, King Abdulaziz University, Jeddah, Saudi Arabia \\ ${ }^{10}$ College of Medicine, King Faisal University, Hofuf, Saudi Arabia \\ ${ }^{11}$ College of Medicine, University of Debrecen, Debrecen, Hungary
}

Received: 20 December 2021

Accepted: 04 January 2022

\section{*Correspondence: \\ Dr. Rahmathulla Safiyul Rahman, \\ E-mail: safiyul@gmail.com}

Copyright: (c) the author(s), publisher and licensee Medip Academy. This is an open-access article distributed under the terms of the Creative Commons Attribution Non-Commercial License, which permits unrestricted non-commercial use, distribution, and reproduction in any medium, provided the original work is properly cited.

\begin{abstract}
Colon polyps might originate from the submucosa including lymphoid aggregates, carcinoids and lipomas. On the other hand, most polyps usually arise from the mucosa and include various types, whether neoplastic or not. The prognosis and treatment of these lesions depend on establishing an adequate diagnosis to rule out the presence of malignancy. Therefore, clinicians should be aware of each subclassification's different types and presentations to achieve the best outcomes. When conducting colonoscopy screening for colorectal cancer, colorectal polyps are commonly discovered. The prevalence of these lesions is high. However, most of them do not have any clinical significance. On the other hand, evidence shows that some polyps might have premalignant characteristics, which are usually challenging to manage in clinical practice. Therefore, evidence shows that the most appropriate approach to managing these lesions and achieving the best prognosis would be identifying and treating them as early as possible before complications appear to intervene against potential morbidities and mortality. Clinicians should consider the wide variations of colorectal cancer to establish the most appropriate diagnosis. A histological diagnosis is essential in these events to exclude malignancy and decide the most appropriate treatment plan.
\end{abstract}

Keywords: Diagnosis, Management, Colorectal carcinoma, Colorectal polyps, Prognosis, Types

\section{INTRODUCTION}

Colorectal polyps are usually discovered during screening for colorectal cancer. However, they might be found as sporadic lesions or part of another syndrome. They can be classified based on their size into large, small or diminutive if their diameter was $\geq 1 \mathrm{~cm}, 6-9 \mathrm{~mm}$ and $\leq 5$ $\mathrm{mm}$, respectively. They can also be pedunculated, sessile, flat or depressed. ${ }^{1}$ Many risk factors were reported in the literature including excess alcohol intake, tobacco use, low-fiber and high-fat diets, male gender and advancing age. Family history and inflammatory bowel diseases 
might also be associated with the epidemiology of colorectal polyps. $^{2}$

It has been furtherly shown that some polyps might originate from the submucosa including lymphoid aggregates, carcinoids, and lipomas. On the other hand, most polyps usually arise from the mucosa and include various types, whether neoplastic or not. The prognosis and treatment of these lesions depend on establishing an adequate diagnosis to rule out the presence of malignancy. ${ }^{3-5}$ Therefore, clinicians should be aware of each subclassification's different types and presentations to achieve the best outcomes. Accordingly, we aimed to conduct the current literature review to discuss colonic polyps' various types, differences and prognosis.

\section{METHODS}

This literature review was based on an extensive literature search in Medline, Cochrane and EMBASE databases which was performed on 27 November 2021 using the medical subject headings (MeSH) or a combination of all possible related terms, according to the database. To avoid missing potential studies, a further manual search for papers was done through Google Scholar while the reference lists of the initially included papers. Papers discussing types, differences and prognosis of colonic polyps were screened for useful information. No limitations were posed on date, language, age of participants or publication type.

\section{DISCUSSION}

Evidence showed various types of colonic polyps and the commonest include adenomatous and hyperplastic types (Figure 1). However, there were also other types of colonic polyps that will be discussed in this section. Therefore, we will divide the current study into sections based on the type of polyps and under each section, we discussed the main features and prognostic outcomes for each type to facilitate understanding.

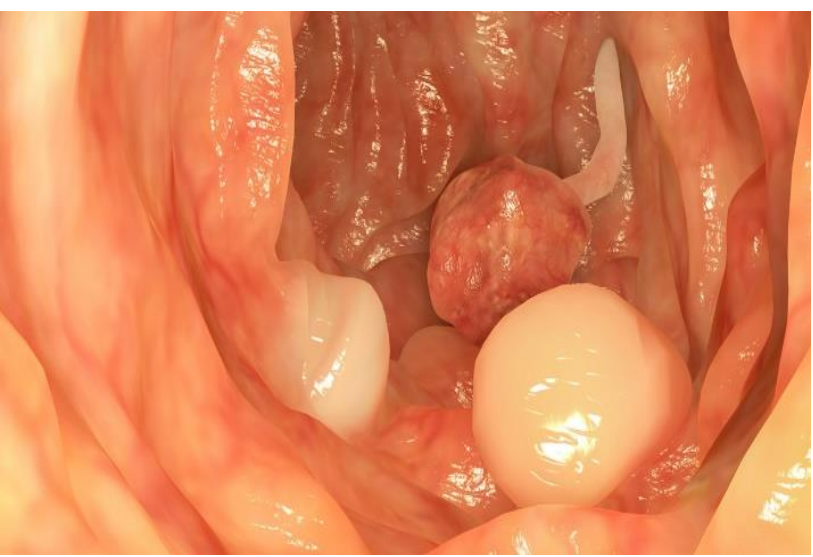

Figure 1: Macroscopic appearance of colorectal polyps.

\section{Hyperplastic polyps}

These colonic polyps were usually sessile, small and localized at the end of the colon (including the sigmoid colon and rectum). Thus, evidence showed that they were not correlated with malignancy and were not worrisome. Moreover, it had been reported that hyperplastic polyps were difficult to differentiate from adenomatous polyps during colonoscopy. However, microscopic examination of these polyps showed hyperplasia without dysplasia (Figure 2). Therefore, they were not considered precancerous lesions. Accordingly, it had been shown that a biopsy was often needed from a hyperplastic polyp to adequately differentiate it from adenomatous polyps and perform adequate treatment. Evidence suggested that hyperplastic polyps that were $>2 \mathrm{~mm}$ in diameter should be removed because they might have a high risk of dysplasia and can be associated with malignant degeneration. In addition, old studies reported that they were considered separate lesions, while recent evidence indicated that they were usually part of starred polyps with no malignant potential. ${ }^{6,7}$

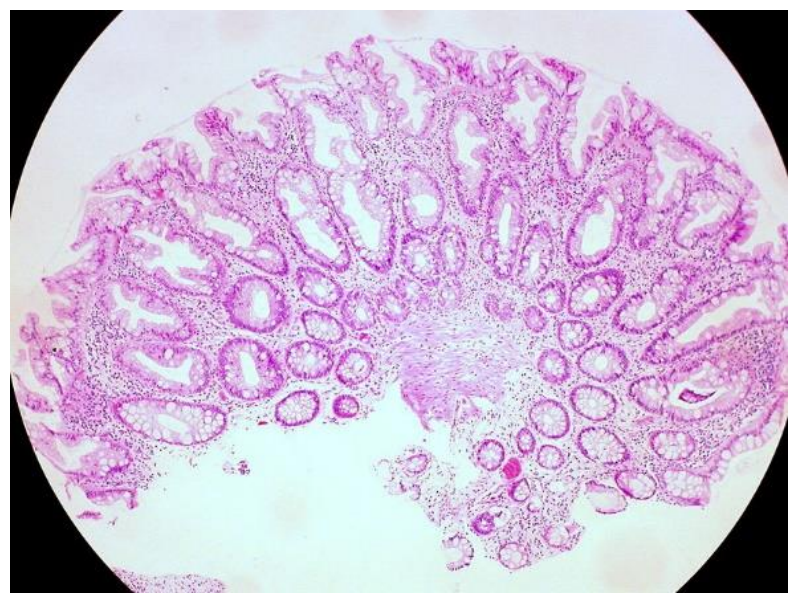

Figure 2: Microscopic picture of a hyperplastic polyp of the rectum.

\section{Inflammatory polyps}

These polyps were also termed pseudopolyps. They were most commonly reported among patients with inflammatory bowel diseases, particularly ulcerative colitis. Ischemic or infectious colitis might also predispose to the development of these polyps. It had been shown that were not usually true polyps. On the other hand, they were formed from distorted mucosal anatomy and accumulations of inflammatory mediators. Fortunately, it had been shown that these lesions were not usually precancerous. However, evidence showed that microscopic examination cannot adequately differentiate them from adenomatous polyps. Accordingly, a biopsy should be done for these polyps to determine a proper diagnosis and management and excluded malignancy. It should be noted that there were no recommendations to perform surgical excision of these polyps unless patients 
suffered from associated clinical manifestations. Microscopic evaluation of these polyps showed islands of regenerating normal mucosa that was usually surrounded by regions of mucosal loss and degeneration. However, extensive polyposis might also be found in some patients, particularly those with severe colitis. In such cases, it had been evidenced that such lesions were hard to differentiate from familial adenomatous polyposis.

\section{Hamartomatous polyps}

These polyps were formed of a connective tissue core including inflammatory infiltrates, lamina propria and smooth muscles, with a hypertrophic epithelium cover. Accordingly, these were usually termed juvenile polyps. This was because most of these polyps (75\%) developed in children $<10$ years of age. Overall, it had been reported that hamartomatous polyps usually developed early, especially the sporadic type. The macroscopic appearance of these polyps showed that they were usually smooth, cherry-red and pedunculated polyps. Moreover, evidence showed that they might be hard to differentiate from pedunculated adenomatous polyps. It was furtherly shown that they might present as a single lesion and be part of another polyposis syndrome. Sporadic polyps were not usually precancerous and did not potentially turn into a malignant lesion. However, it had been shown that bleeding might be a severe complication because they were highly vascularized. Other complications might also include intestinal obstruction and intussusception. Finally, multiple polyps tended to be part of genetic polyposis syndromes. ${ }^{8,9}$

\section{Submucosal colorectal lesions}

A differential diagnosis should be conducted with these lesions (whether malignant or benign) to differentiate them from colorectal polyps. Many benign lesions were reported including endometriosis, hemangiomas, pneumatosis cystoides intestinalis, isolated lymphoid nodules and lipomas were typical examples of benign lesions. On the other hand, it had been shown that lymphomas, gastrointestinal stromal tumors (GISTs), metastasis, carcinoid tumors and premalignant polyps can be considered malignant lesions. ${ }^{10}$ Therefore, it was critical to establish an adequate diagnosis of these lesions. Moreover, it had been shown that when a biopsy failed to determine the underlying etiology, computed tomography can be approached to establish a proper diagnosis. In addition, endorectal ultrasonography (ERUS) and ERUSguided biopsy can also be performed to diagnose rectal lesions.

\section{Adenomatous polyps}

Estimates showed that adenomas represent around twothirds of the colonic polyps. Unfortunately, reports showed that these polyps might potentially turn into cancer. However, most of them were benign and did not develop into cancer. Specific microscopic characteristics, general appearance and size were useful in determining the classification of adenomatous polyps. It had been ruled out that the risk of developing cancer from adenomatous polyps was significantly correlated with the size of the polyp. The larger the size of the polyp, the higher the risk it would develop into cancer. Accordingly, it had been shown that large polyps $(>5 \mathrm{~mm})$ should be removed to intervene against cancer development. Moreover, a microscopic evaluation should also be conducted to assess the prognosis and provide adequate follow up for these cases. A previous case series also demonstrated that gender and age were important factors determining the rate of adenomas. ${ }^{11} \mathrm{~A}$ previous population-based investigation demonstrated that adenomatous polyps were discovered among asymptomatic patients undergoing colorectal screening in $34.3 \%$ of them. ${ }^{12}$ It had been further demonstrated that autopsy-based investigations showed that the prevalence was even higher among asymptomatic patients. ${ }^{13}$ Many factors attributed to the development of these lesions. The most important factors included environmental factors and genetic susceptibility. Smoking was also a significant risk factor for developing these lesions. ${ }^{14,15}$ Other risk factors might also include low intake of calcium and fibers, high intake of meats and obesity. ${ }^{16,17}$ On the other hand, some studies reported that the administration of statins and non-steroidal anti-inflammatory medications might be protective against the development of these lesions. ${ }^{17,18}$

The distribution of these adenomatous polyps had been found to occur in any part of the colon and large polyps have a similar distribution to carcinomas. Therefore, colonoscopy was usually diagnostic and most patients usually presented with symptoms. However, intestinal obstruction and hematochezia might be potential for adenomatous polyps that might lead to other diagnostic manifestations. Moreover, villous adenoma was usually associated with hypokalemia and secretory diarrhea (McKittrick-Wheelock syndrome). Histological examination of adenomatous polyps can classify them into villous, tubular or tubulovillous adenomas. Tubular polyps were the most commonly reported and included around $80 \%$ of the whole polyps. The histological type and adenoma size were the main dependant risk factors for developing carcinoma. Overall, it had been shown that the risk of carcinoma might be $50 \%$ in $>2 \mathrm{~cm}$ villous adenomas and $10 \%$ in $<1 \mathrm{~cm}$ tubular adenomas, while an intermediate risk $(22 \%)$ was reported for tubulovillous adenomas. ${ }^{19}$ On the other hand, a previous investigation by Bertelson et al demonstrated that neither size nor histological appearance correlated with developing cancer. $^{20}$

\section{Malignant polyps}

Malignant polyps usually referred to polyps that contain malignant cells. Various factors including microscopic examination and the extent of cancer were usually used to determine the optimal management approach for these 
polyps. As previously mentioned, adenomatous polyps were the most significant precancerous lesions and the risk was dependant on the size and histological pattern of the polyp. ${ }^{21,22}$ Therefore, an adequate diagnosis should be provided for cancerous lesions to plan a proper treatment and follow up strategy and enhance the prognosis of the affected patients. Evidence showed that carcinomas that did not extend beyond mascularis mucosae did not usually have metastatic features. Therefore, local excision was usually adequate treatment. On the other hand, metastasis was reported if muscularis mucosa infiltrated. In such cases, simple local excision might not be adequate for proper management. Accordingly, in the previous investigation by Haggitt et al the authors developed a classification system upon which surgeons can decide whether a wider excision might be needed for polyps containing cancer. ${ }^{23}$ The criteria were presented in Figure 3. All of them should be found to demonstrate that polypectomy was enough management approach. However, the authors also reported some findings or criteria that should indicate the non-adequacy of polypectomy for polyps containing cancer (Figure 4). This was because there might be a risk of lymph node metastasis $(10 \%)$. In these events, surgical resection was usually the recommended management modality. Therefore, establishing a proper diagnosis and evaluation of the lesion was mandatory to determine the most appropriate management approach. The detailed diagnosis and treatment of polyps containing cancer were described elsewhere in the literature and were not included within the scope of the current review. ${ }^{19}$

\section{- The polyp must be pedunculated}

- The tumor does not extend beyond the head or neck of the polyp (Haggitt's levels 1 and 2, respectively)

- The distance between the tumor edge and the margin of the specimen exceeds $2 \mathrm{~mm}$

- The histology is favorable (not poorly differentiated and no lymphatic or vascular invasion)

Figure 3: Criteria indicating the adequacy of polypectomy for polyps containing cancer. ${ }^{23}$

- The polyp is sessile

- A pedunculated polyp is identified with invasion of the tumor to any part of its stalk beneath

its neck or to the submucosa of the bowel wall beneath the stalk (Haggitt's levels 3 and 4, respectively)

- The distance between the tumor edge and the margin of the specimen is less than $2 \mathrm{~mm}$

- The histology is poorly differentiated

- Lymphatic or vascular invasion is observed

Figure 4: Criteria indicating the non-adequacy of polypectomy for polyps containing cancer. ${ }^{23}$

\section{Starred adenomas and polyposis syndromes}

Starred polyps were previously identified as hyperplastic polyps. However, recent evidence differentiated them from benign and malignant polyps as they were usually precancerous. Starred adenomas were so-called because of their histological appearance. They represented a heterogeneous group of polyps and were also called sessile serrated adenomas. They occured more commonly in women and evidence also showed that they developed in the right colon. ${ }^{24}$ It had been furtherly shown that advanced synchronous neoplasia was usually associated with large serrated polyps. Therefore, adequate diagnosis and follow up should be conducted for these patients to intervene against the turnover into a malignant lesion. ${ }^{6,24,25}$
In another context, many polyposis syndromes were reported in the literature. The commonest one included familial adenomatous polyposis. Evidence showed that polyps in these syndromes were associated with other gastrointestinal manifestations and were usually responsible for $1 \%$ of the total colorectal cancer. In addition, it had been furtherly shown that extraintestinal tumors might also be present in the affected patients. ${ }^{26}$ Other syndromes reported in the literature included PeutzJeghers syndrome, MUTYH-associated polyposis syndrome, familial juvenile polyposis syndrome, serrated polyposis syndrome, hereditary mixed polyposis syndrome, Cronkite-Canada syndrome and PTEN hamartomatous tumor syndromes. Genetic predisposition was usually present in most of these syndromes in addition to the different environmental risk factors. 
Therefore, genetic analysis was usually required for establishing a better diagnosis. Moreover, a systemic evaluation of patients should be conducted because most of them also have extraintestinal manifestations, which might affect the overall prognosis of the affected patients. ${ }^{19}$

\section{CONCLUSION}

When conducting colonoscopy screening for colorectal cancer, colorectal polyps are commonly discovered. The prevalence of these lesions is high. However, most of them do not have any clinical significance. On the other hand, evidence shows that some polyps might have premalignant characteristics, which are usually challenging to manage in clinical practice. Therefore, evidence shows that the most appropriate approach to managing these lesions and achieving the best prognosis would be identifying and treating them as early as possible before complications appear to intervene against potential morbidities and mortality. Clinicians should consider the wide variations of colorectal cancer to establish the most appropriate diagnosis. A histological diagnosis is essential in these events to exclude malignancy and decide the most appropriate treatment plan.

\section{Funding: No funding sources \\ Conflict of interest: None declared \\ Ethical approval: Not required}

\section{REFERENCES}

1. Meseeha M, Attia M. Colon Polyps. Treasure Island (FL): StatPearls Publishing; 2021.

2. Yoshizawa N, Yamaguchi H, Kaminishi M. Differential diagnosis of solitary gastric PeutzJeghers-type polyp with stomach cancer: a case report. Int J Surg Case Rep. 2018;51:261-4.

3. Turner JS, Henry D, Chase A, Kpodzo D, Flood MC, Clark CE. Adenoma detection rate in colonoscopy: does the participation of a resident matter? Am Surgeon. 2018;84(6):1064-8.

4. Hsieh YH, Leung FW. Increase your adenoma detection rate without using fancy adjunct tools. Tzu-Chi Med J. 2018;30(3):127-34.

5. Chen EY, Vaccaro GM. Small bowel adenocarcinoma. Clinic Colon Rect Surg. 2018;31(5):267-77.

6. Sweetser S, Smyrk TC, Sinicrope FA. Serrated colon polyps as precursors to colorectal cancer. Clinic Gastroenterol Hepatol. 2013;11(7):760-7.

7. Son PT, Reda A, Viet DC, Quynh NXT, Hung DT, Tung TH, et al. Exchange transfusion in the management of critical pertussis in young infants: a case series. Vox Sanguinis. 2021;116(9):976-82.

8. El-Qushayri AE, Dahy A, Reda A, Mahmoud MA, Mageed SA, Kamel AMA, et al. A closer look to the high burden of the psychiatric disorders among health care workers (HCWs) in Egypt during
COVID-19 outbreak: a meta-analysis of 3137 HCWs. Epidemiol Health. 2021;43:2021045.

9. El-Qushayri AE, Ghozy S, Reda A, Kamel AMA, Abbas AS, Dmytriw AA. The impact of Parkinson's disease on manifestations and outcomes of Covid-19 patients: a systematic review and meta-analysis. Rev Med Virol. 2021:2278.

10. Nguyen TM, Huan VT, Reda A, Morsy S, Giang HTN, Tri VD, et al. Clinical features and outcomes of neonatal dengue at the Children's Hospital 1, Ho Chi Minh, Vietnam. J Clinic Virol. 2021;138:104758.

11. Diamond SJ, Enestvedt BK, Jiang Z, Holub JL, Gupta M, Lieberman DA, et al. Adenoma detection rate increases with each decade of life after 50 years of age. Gastrointest Endosc. 2011;74(1):135-40.

12. Reinhart K, Bannert C, Dunkler D, Salzl P, Trauner $\mathrm{M}$, Renner F, et al. Prevalence of flat lesions in a large screening population and their role in colonoscopy quality improvement. Endoscopy. 2013;45(5):350-6.

13. O'Brien MJ, Winawer SJ, Zauber AG, Gottlieb LS, Sternberg SS, Diaz B, et al. The National Polyp Study. Patient and polyp characteristics associated with high-grade dysplasia in colorectal adenomas. Gastroenterology. 1990;98(2):371-9.

14. Burnett-Hartman AN, Passarelli MN, Adams SV, Upton MP, Zhu L, Potter JD, et al. Differences in epidemiologic risk factors for colorectal adenomas and serrated polyps by lesion severity and anatomical site. Am J Epidemiol. 2013;177(7):625-37.

15. Thieu H, Dat B, Nam NH, Reda A, Duc NT, Alshareef A, et al. Antibiotic resistance of Helicobacter pylori infection in a children's hospital in Vietnam: prevalence and associated factors. Minerva Medica. 2020;111(5):498-501.

16. Okabayashi K, Ashrafian H, Hasegawa H, Yoo J, Patel VM, Harling L, et al. Body mass index category as a risk factor for colorectal adenomas: a systematic review and meta-analysis. Am J Gastroenterol. 2012;107(8):1175-85.

17. Fu Z, Shrubsole MJ, Smalley WE, Wu H, Chen Z, Shyr Y, et al. Lifestyle factors and their combined impact on the risk of colorectal polyps. Am J Epidemiol. 2012;176(9):766-76.

18. Broughton T, Sington J, Beales IL. Statin use is associated with a reduced incidence of colorectal adenomatous polyps. Int $\mathrm{J}$ Colorect Dis. 2013;28(4):469-76.

19. Shussman N, Wexner SD. Colorectal polyps and polyposis syndromes. Gastroenterol Rep. 2014;2(1):1-15.

20. Bertelson NL, Kalkbrenner KA, Merchea A, Dozois EJ, Landmann RG, Petris GD, et al. Colectomy for endoscopically unresectable polyps: how often is it cancer? Dise Colon Rect. 2012;55(11):1111-6.

21. Sakamoto T, Matsuda T, Nakajima T, Saito Y. Clinicopathological features of colorectal polyps: 
evaluation of the 'predict, resect and discard' strategies. Colorect Dis. 2013;15(6):295-300.

22. Dibas M, Doheim MF, Ghozy S, Ros MH, El-Helw GO, Reda A. Incidence and survival rates and trends of skull base chondrosarcoma: a population-based study. Clinic Neurol Neurosurg. 2020;198:106153.

23. Haggitt RC, Glotzbach RE, Soffer EE, Wruble LD. Prognostic factors in colorectal carcinomas arising in adenomas: implications for lesions removed by endoscopic polypectomy. Gastroenterology. 1985;89(2):328-36.

24. Sugumar A, Sinicrope FA. Serrated polyps of the colon. F1000 Med Rep. 2010;2:89.

25. Winawer S, Fletcher R, Rex D, Bond J, Burt R, Ferrucci $\mathrm{J}$, et al. Colorectal cancer screening and surveillance: clinical guidelines and rationaleUpdate based on new evidence. Gastroenterology. 2003;124(2):544-60.

26. Aretz S. The differential diagnosis and surveillance of hereditary gastrointestinal polyposis syndromes. Dtsch Arztebl Int. 2010;107(10):163-73.

Cite this article as: Rahman RS, Alharbi AS, Basaben BA, Alsalman AA, Aljohani AS, Banafea $\mathrm{BA}$, et al. Types, differences and prognosis of colonic polyps. Int J Community Med Public Health 2022;9:1034-9. 